\title{
Optimizing Quantize-Map-and-Forward Relaying for Gaussian Diamond Networks
}

\author{
Ayan Sengupta, I-Hsiang Wang, Christina Fragouli \\ École Polytechnique Fédérale de Lausanne (EPFL), Lausanne, Switzerland \\ Email: \{ayan.sengupta, i-hsiang.wang, christina.fragouli\}@epfl.ch
}

\begin{abstract}
We evaluate the information-theoretic achievable rates of Quantize-Map-and-Forward (QMF) relaying schemes over Gaussian $N$-relay diamond networks. Focusing on vector Gaussian quantization at the relays, our goal is to understand how close to the cutset upper bound these schemes can achieve in the context of diamond networks, and how much benefit is obtained by optimizing the quantizer distortions at the relays. First, with noise-level quantization, we point out that the worst-case gap from the cutset upper bound is $\left(N+\log _{2} N\right)$ bits/s/Hz. A better universal quantization level found without using channel state information (CSI) leads to a sharpened gap of $\log _{2} N+\log _{2}(1+N)+N \log _{2}(1+1 / N)$ bits/s/Hz. On the other hand, it turns out that finding the optimal distortion levels depending on the channel gains is a non-trivial problem in the general $N$-relay setup. We manage to solve the two-relay problem and the symmetric $N$-relay problem analytically, and show the improvement via numerical evaluations both in static as well as slow-fading channels.
\end{abstract}

\section{INTRODUCTION}

Quantize-Map-and-Forward (QMF) was the first proposed relaying strategy that was proved to achieve the capacity of arbitrary Gaussian networks within a bounded gap [1]. Its merit as a breakthrough approach is not questioned; it is evidenced by the interest it created and the number of other works it inspired. However, what can be questioned is its practicality: a recurrent criticism in the community is that the bounded gap from capacity $(15 M \mathrm{bits} / \mathrm{sec} / \mathrm{Hz}$ in [1] improved to $1.26 M$ in [3], with $M=N+2$ being the number of nodes in the unicast network and $N$ being the number of relays) makes this more a result of theoretical interest, rather than a strategy that could be exploited for practical deployments. Opposing this criticism is the observation that the gap proved in [1], [2], [3] is worst-case-the actual gap of the scheme from the cutset bound might in practice be smaller. Moreover, the proposed QMF schemes in [1], [2] and [3] for Gaussian networks use the same quantizer on all relay nodes, which does not depend on the channel parameters. Could CSI-aware optimization of individual quantizers at each relay further sharpen the gap?

To answer the above questions, we evaluate the performance of QMF over specific small networks that may be interesting from a practical viewpoint. The first class of networks where, in our opinion, we may see deployments of such ideas is the $N$-relay diamond network (see Fig. 1). We initially study the

The work was supported by the EU project CONECT FP7-ICT-2009257616 and the ERC Starting Grant project NOWIRE ERC-2009-StG-240317. information-theoretic achievable rates of QMF with noise-level quantization at all relays (as in [1], [2]) in such networks with full-duplex relays, and determine how far they are from the cutset upper bound. Furthermore, focusing QMF relaying on this class of relay networks, we optimize the achievable rate by finding the best quantization distortion level at each relay, and study how much improvement such optimizations can provide. We find that, for the $N$-relay network and the noiselevel quantization scheme, the worst-case gap to the cutset bound is $\left(N+\log _{2} N\right)$ bits/s/Hz, which is an improvement (for this class of networks) over the best previous known bound of $1.26(N+2)$ bits $/ \mathrm{s} / \mathrm{Hz}$ [3]. We show that following the reasoning in [3], a better universal quantizer can be found by optimizing the worst-case gap between the QMF achievable rate and the cutset bound. Without using CSI, the worst-case gap is sharpened to $\log _{2} N+\log _{2}(1+N)+N \log _{2}(1+1 / N)$ bits/s/Hz. On the other hand, it turns out that CSI-aware quantizer optimization is non-trivial for the general $N$-relay setup. We completely characterize the optimal choice for the case of 2 relays, as well the case of $N$-relays with a symmetrical structure where the channels in the broadcast part and the multiple-access part of the network are identical (but can differ between the broadcast and multiple-access parts).

For the CSI-aware quantizer-optimized scheme, we consistently observe a much smaller gap in our evaluation. For the two-relay case, the gap is reduced to less than $2 \mathrm{bits} / \mathrm{s} / \mathrm{Hz}$, as opposed to the worst-case gap of $3 \mathrm{bits} / \mathrm{s} / \mathrm{Hz}$ in the noiselevel quantization scheme. The improvements are even more pronounced when there are more than two relays.

Looking deeper into the performance of the two-relay network, we find that the worst-case gap of QMF occurs mostly for configurations where the multiple access cut becomes the min-cut; the main reason being that QMF does not provide the coherent-combining power gain in this setting. An alternative is to use decode-and-forward relays, since the relays are closer to the source than to the destination.

Our evaluations over slow-fading channels also show similar improvements with quantizer optimization, where the gap to $1 \%$-outage capacity is roughly $0.5 \mathrm{bit} / \mathrm{s} / \mathrm{Hz}$ in the symmetrical Rayleigh-faded scenario.

In the literature, the best known result for the capacity of the two-relay (static) Gaussian diamond network is due to [1], where it is proved that partial decode-and-forward can achieve the capacity to within $1 \mathrm{bit} / \mathrm{s} / \mathrm{Hz}$. On the other hand, for the 
$N$-relay network, if the network has a symmetrical structure, it is shown that amplify-and-forward can achieve the capacity to within $3.6 \mathrm{bits} / \mathrm{s} / \mathrm{Hz}$ [4]. However, in fading wireless channels, the above two schemes are not very practical: the partial decode-and-forward scheme requires the source to have global instantaneous CSI of the network, while the amplify-andforward scheme requires the phases of the forward channels.

Therefore, although the above schemes may outperform QMF in specific static channel configurations, QMF indeed does better over fading wireless channels [6], since with noiselevel quantization, QMF does not require forward CSI at the relays or global CSI at the source. Even for the quantizeroptimized QMF, only the channel strengths of forward channels are required at the relays.

The rest of this paper is organized as follows: We first formulate the problem in Section II, evaluate the QMF achievable rate with universal quantization and derive the gap results. Next, in Section III, we present the CSI-aware quantizer-optimized QMF scheme and solve the optimization problem for the two-relay case and the symmetrical $N$-relay case. Finally, Section IV offers numerical evaluations of the proposed schemes.

\section{SySTEM MOdEL AND FORMUlation}

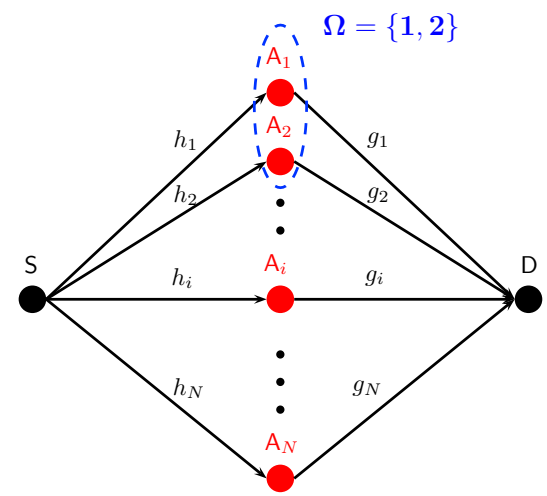

Fig. 1. The $N$-Relay Diamond Network with a partition $\Omega=\{1,2\}$ on the set of relays. In the nomenclature considered in the paper, the cut corresponding to the shown $\Omega$ is $\left\{\mathrm{S}, \mathrm{A}_{1}, \mathrm{~A}_{2}\right\}$.

\section{A. Communication Scenario}

The $N$-Relay diamond network we consider in this paper is shown in Fig. 1. In this model, the source $S$ communicates with the destination $\mathrm{D}$ via $N$ relay nodes. The signals transmitted by the source $S$ and the relay nodes $A_{i} \in\left\{A_{1}, A_{2}, \ldots, A_{N}\right\}$ are denoted by $X$ and $X_{i}$. The received signal at the destination and the relay nodes are denoted by $Y$ and $Y_{i}$, $i \in\{1,2, \ldots, N\}$. The relays are capable of causal signal processing. The received signals as a function of the transmitted signals are as follows:

$$
Y_{i}=h_{i} X+Z_{i}, Y=\sum_{i=1}^{N} g_{i} X_{i}+Z
$$

where $h_{i}$ represents the complex channel coefficient between $\mathrm{S}$ and $\mathrm{A}_{i}$ and $g_{i}$ denotes that between $\mathrm{A}_{i}$ and D. $Z_{i}, i \in$ $\{1,2, \ldots, N\}$ and $Z$ are i.i.d $\mathcal{C N}(0,1)$ random variables. The transmitted signals are normalized to have an average power constraint of unity at the source and the relays, i.e., $\mathbb{E}\left(|X|^{2}\right) \leq$ 1 and $\mathbb{E}\left(\left|X_{i}\right|^{2}\right) \leq 1, i \in\{1,2, \ldots, N\}$.

\section{B. Cutset Upper Bound on Capacity}

We denote by $\Omega$, a partition of the index set $[1: N]:=$ $\{1,2, \ldots, N\}$ of the relay nodes $\left\{\mathrm{A}_{1}, \ldots, \mathrm{A}_{N}\right\}$. Therefore, for any $\Omega \subseteq[1: N],\{\mathrm{S}\} \cup\left\{\mathrm{A}_{i}: i \in \Omega\right\}$ is a cut of the network (see Fig. 1 for illustration). Moreover, let $X_{\Omega}:=\left\{X_{i}: i \in \Omega\right\}$.

The cutset upper bound on the capacity of the network is given by (1) in the following. For the purpose of gap evaluation, we further upper bound it by exchanging the max and min signs as follows: ${ }^{1}$ (all logarithms are to the base 2)

$$
\begin{aligned}
& \bar{C}_{c u t}=\max _{p\left(X, X_{[1: N]}\right)} \min _{\Omega} I\left(X, X_{\Omega} ; Y_{\Omega^{c}}, Y \mid X_{\Omega^{c}}\right) \\
& \leq \min _{\Omega} \max _{p\left(X, X_{[1: N]}\right)} I\left(X, X_{\Omega} ; Y_{\Omega^{c}}, Y \mid X_{\Omega^{c}}\right) \\
& =\min _{\Omega}\left\{\log \left(1+\left(\sum_{i \in \Omega}\left|g_{i}\right|\right)^{2}\right)+\log \left(1+\sum_{j \in \Omega^{c}}\left|h_{j}\right|^{2}\right)\right\}
\end{aligned}
$$

\section{QMF Achievable Rates with Universal Quantizers}

The achievable rate for QMF relaying over the $N$-relay diamond network is evaluated using the single-letter characterization $^{2}$ in [3].

$$
R_{\mathrm{QMF}}=\max \min _{\Omega}\left\{\begin{array}{l}
I\left(X, X_{\Omega} ; \widehat{Y}_{\Omega^{c}}, Y \mid X_{\Omega^{c}}\right) \\
-I\left(Y_{\Omega} ; \widehat{Y}_{\Omega} \mid X, X_{[1: N]}, \widehat{Y}_{\Omega^{c}}, Y\right)
\end{array}\right\}
$$

where the maximum is taken over all probability distribution $p(X) \prod_{i \in[1: N]} p\left(X_{i}\right) p\left(\widehat{Y}_{i} \mid Y_{i}, X_{i}\right)$. Here $\widehat{Y}_{i}$ denotes the quantizer output of relay $\mathrm{A}_{i}$. Generating the Gaussian vector quantization codebook with the following single-letter probability distribution $\widehat{Y}_{i}=Y_{i}+\widehat{Z}_{i}, \widehat{Z}_{i} \sim \mathcal{C N}\left(0, \Delta_{i}\right), i=1, \ldots, N$, the above achievable rate is evaluated as

$$
R_{\mathrm{QMF}, \mathrm{G}}\left(\Delta_{[1: N]}\right)=\min _{\Omega}\left\{R\left(\Omega ; \Delta_{[1: N]}\right)\right\}
$$

where $\Delta_{J}:=\left\{\Delta_{j} \mid j \in J\right\}$, and $R\left(\Omega ; \Delta_{[1: N]}\right)$ is as follows:

$$
\begin{aligned}
R & \left(\Omega ; \Delta_{[1: N]}\right) \\
= & I\left(X, X_{\Omega} ; \widehat{Y}_{\Omega^{c}}, Y \mid X_{\Omega^{c}}\right)-I\left(Y_{\Omega} ; \widehat{Y}_{\Omega} \mid X, X_{[1: N]}, \widehat{Y}_{\Omega^{c}}, Y\right) \\
= & h\left(Y \mid X_{\Omega^{c}}\right)+h\left(\widehat{Y}_{\Omega^{c}}\right)-h\left(\widehat{Y}_{\Omega^{c}} \mid X\right)-h(Z) \\
& -h\left(\widehat{Y}_{\Omega} \mid X\right)+h\left(\widehat{Y}_{\Omega} \mid Y_{\Omega}\right) \\
= & {\left[\begin{array}{l}
\log \left(1+\sum_{i \in \Omega}\left|g_{i}\right|^{2}\right)+\log \left(1+\sum_{j \in \Omega^{c}} \frac{\left|h_{j}\right|^{2}}{1+\Delta_{j}}\right) \\
-\sum_{i \in \Omega} \log \left(\frac{1+\Delta_{i}}{\Delta_{i}}\right)
\end{array}\right]^{+} }
\end{aligned}
$$

Comparing the achievable rate (3) to the upper bound (2), one can observe that the difference lies in

\footnotetext{
${ }^{1}$ Numerical evaluation of (1) shows that there is no significant gap from the relaxed version in (2) for the range of $N$ considered in this paper.

${ }^{2}$ The Gaussian version was proved in [2] using lattice vector quantizers.
} 
1) the coherent combining power gain of the transmitting terminals in $\Omega$

2) the quantization loss at the transmitting terminals in $\Omega$ (the term: $-\sum_{i \in \Omega} \log \left(\frac{1+\Delta_{i}}{\Delta_{i}}\right)$ )

3) the quantization loss at the receiving terminals in $\Omega^{c}$.

Setting the quantizer distortions to be the same as the noise variance, i.e., $\Delta_{i}=1 \forall i \in\{1,2, \ldots, N\}$, we essentially recover the noise-level quantization scheme proposed in [1] and the following achievable rate:

$$
\begin{aligned}
& R\left(\Omega ; \Delta_{[1: N]}=(1, \ldots, 1)\right) \\
& =\left[\log \left(1+\sum_{i \in \Omega}\left|g_{i}\right|^{2}\right)+\log \left(1+\sum_{j \in \Omega^{c}} \frac{\left|h_{j}\right|^{2}}{2}\right)-|\Omega|\right]^{+}
\end{aligned}
$$

Comparing it with the upper bound (2), we see that the gap is upper bounded by $\left(N+\log _{2} N\right)$ bits/s/Hz.

Following the reasoning in [3], we can further obtain a better universal quantizer distortion (in the sense of worstcase gap) that does not depend on channel coefficients. Setting the quantizer distortions to be the same as $\Delta$, we have the following achievable rate:

$$
\begin{aligned}
& R\left(\Omega ; \Delta_{[1: N]}=(\Delta, \ldots, \Delta)\right) \\
& =\left[\begin{array}{l}
\left.\log \left(1+\sum_{i \in \Omega}\left|g_{i}\right|^{2}\right)+\log \left(1+\sum_{j \in \Omega^{c}} \frac{\left|h_{j}\right|^{2}}{1+\Delta}\right)\right]^{+} \\
-|\Omega| \log \frac{1+\Delta}{\Delta}
\end{array}\right]
\end{aligned}
$$

With a term-by-term comparison with the upper bound (2), we see that the worst-case gap is upper bounded by

$$
\operatorname{gap}^{*}(\Delta ; N)=\log N+\log (1+\Delta)+N \log \frac{1+\Delta}{\Delta}
$$

For each $N$, we find $\Delta^{*}:=\arg \min _{\Delta>0} \operatorname{gap}^{*}(\Delta ; N)=N$ by simply taking the derivative of $\operatorname{gap}^{*}(\Delta ; N)$ with respect to $\Delta$. Hence $\operatorname{gap}^{*}\left(\Delta^{*} ; N\right)=\log N+\log (1+N)+N \log \frac{1+N}{N}$ is the worst-case gap using the universal quantizer distortion $\Delta^{*}=N$.

\section{CSI-AWARE QUANTIZER OPTIMIZATION}

If the relays have access to the global CSI of the channel gains (the phases of the channel coefficients are not required), there is scope to optimize the quantizer distortions at the relays. The optimization problem can be stated as:

$$
\begin{aligned}
R_{\mathrm{QMF}, \mathrm{G}}^{*} & =\max _{\Delta_{[1: N]} \geq 0} R_{\mathrm{QMF}, \mathrm{G}}\left(\Delta_{[1: N]}\right) \\
& =\max _{\Delta_{[1: N]} \geq 0} \min _{\Omega}\left\{R\left(\Omega ; \Delta_{[1: N]}\right)\right\}
\end{aligned}
$$

We note that this optimization problem is not convex, as within the minimization part of (4), for $\Omega=\emptyset$, the function $R\left(\Omega ; \Delta_{[1: N]}\right)$ is not concave in $\Delta_{[1: N]}$. Instead, we provide an analytical characterization of the optimizing distortions and the corresponding achievable rates for the 2-relay network, and for the special class of symmetric $N$-Relay networks.

In the remainder, since the phases of the channel gains do not affect the optimization solution, we assume without loss of generality that they are all real, i.e, $h_{i}, g_{i} \in \mathbb{R}, \forall i \in[1: N]$.

\section{A. Solution for the 2-Relay Network}

In order to solve the optimization problem in (4) for the case $N=2$, we consider the following equivalent formulation:

$$
\begin{aligned}
R_{\mathrm{QMF}, \mathrm{G}}^{*} & =\max _{\Delta_{2}>0}\left\{\max _{\Delta_{1}>0} \min _{\Omega} R\left(\Omega ; \Delta_{1}, \Delta_{2}\right)\right\} \\
& :=\max _{\Delta_{2}>0} R_{\mathrm{QMF}, \mathrm{G}}^{*}\left(\Delta_{2}\right)
\end{aligned}
$$

with $R_{\mathrm{QMF}, \mathrm{G}}^{*}\left(\Delta_{2}\right)=\max _{\Delta_{1}>0} \min _{\Omega} R\left(\Omega ; \Delta_{1}, \Delta_{2}\right), \Delta_{2}>0$.

We shall first characterize $R_{\mathrm{QMF}, \mathrm{G}}^{*}\left(\Delta_{2}\right)$ and then optimize it over $\Delta_{2}$ to obtain the solution. The following lemma and theorem summarize the main result.

Lemma 1 (Characterization of $R_{\mathrm{QMF}, \mathrm{G}}^{*}\left(\Delta_{2}\right)$ ): Let us define the following intervals of $\Delta_{2}$ :

$$
\mathcal{I}_{1}:=\left(0, \delta_{1}\right) \quad \mathcal{I}_{2}:=\left[\delta_{1}, \delta_{2}\right) \quad \mathcal{I}_{3}:=\left[\delta_{2}, \infty\right)
$$

where $\delta_{1}:=\frac{\left(1+g_{1}^{2}+g_{2}^{2}\right)\left(1+h_{1}^{2}+h_{2}^{2}\right)+\left(1+g_{2}^{2}\right) h_{1}^{2} h_{2}^{2}}{g_{2}^{2}\left(1+g_{1}^{2}+g_{2}^{2}\right)\left(1+h_{1}^{2}\right)}$, and $\delta_{2}:=$ $\frac{\left(1+g_{1}^{2}\right)\left(1+h_{2}^{2}\right)}{g_{2}^{2}}$. In each range of $\Delta_{2}$, the optimizing $\Delta_{1}^{*}$ and $\Omega^{*}$ in the max-min problem $\max _{\Delta_{1}>0} \min _{\Omega} R\left(\Omega ; \Delta_{1}, \Delta_{2}\right)$ is given as follows:

- $\Delta_{2} \in \mathcal{I}_{1}$ :

$$
\Delta_{1}^{*}=\frac{\left(1+g_{2}^{2}\right)\left(1+h_{1}^{2}\right)}{g_{1}^{2}} \quad \Omega^{*}=\{1,2\} \text { or }\{2\}
$$

- $\Delta_{2} \in \mathcal{I}_{2}$ :

$$
\Delta_{1}^{*}=\frac{\left(1+h_{1}^{2}\right) \Delta_{2}+\left(1+h_{1}^{2}+h_{2}^{2}\right)}{\left(g_{1}^{2}+g_{2}^{2}\right) \Delta_{2}-\left(1+h_{2}^{2}\right)} \quad \Omega^{*}=\{1,2\} \text { or } \emptyset
$$

- $\Delta_{2} \in \mathcal{I}_{3}$ :

$$
\Delta_{1}^{*}=\frac{\left(1+h_{1}^{2}\right) \Delta_{2}+\left(1+h_{1}^{2}+h_{2}^{2}\right)}{g_{1}^{2}\left(\Delta_{2}+\left(1+h_{2}^{2}\right)\right)} \quad \Omega^{*}=\{1\} \text { or } \emptyset
$$

Moreover we always have $0<\delta_{1}<\delta_{2}$, and hence the three intervals $\mathcal{I}_{1}, \mathcal{I}_{2}, \mathcal{I}_{3}$ are not empty.

Proof: See Appendix A

Theorem 1: Let $A:=h_{1}^{2}\left(1+h_{1}^{2}\right)-h_{2}^{2}\left(1+h_{1}^{2}+g_{1}^{2}+g_{2}^{2}\right)$, $B:=2 h_{1}^{2}\left(1+h_{1}^{2}\right), C:=h_{1}^{2}\left(1+h_{1}^{2}+h_{2}^{2}\right)$, and $\delta_{3}:=$ $\frac{-B-\sqrt{B^{2}-4 A C}}{2 A}$.

The solution to the maximization problem in (5), is summarized below:

1) $A \geq 0$ or $\delta_{3} \in \mathcal{I}_{3}$ : The optimal solution is

$$
\begin{aligned}
\Delta_{2}^{*}=\delta_{2} \quad \Delta_{1}^{*} & =\frac{\left(1+h_{1}^{2}\right) \delta_{2}+\left(1+h_{1}^{2}+h_{2}^{2}\right)}{\left(g_{1}^{2}+g_{2}^{2}\right) \delta_{2}-\left(1+h_{2}^{2}\right)} \\
& =\frac{\left(1+h_{1}^{2}\right) \delta_{2}+\left(1+h_{1}^{2}+h_{2}^{2}\right)}{g_{1}^{2}\left(\delta_{2}+\left(1+h_{2}^{2}\right)\right)}
\end{aligned}
$$

2) $A<0$ and $\delta_{3} \in \mathcal{I}_{1}$ : The optimal solution is

$$
\begin{aligned}
\Delta_{2}^{*}=\delta_{1} \quad \Delta_{1}^{*} & =\frac{\left(1+h_{1}^{2}\right) \delta_{1}+\left(1+h_{1}^{2}+h_{2}^{2}\right)}{\left(g_{1}^{2}+g_{2}^{2}\right) \delta_{1}-\left(1+h_{2}^{2}\right)} \\
& =\frac{\left(1+g_{2}^{2}\right)\left(1+h_{1}^{2}\right)}{g_{1}^{2}}
\end{aligned}
$$

3) $A<0$ and $\delta_{3} \in \mathcal{I}_{2}$ : The optimal solution is

$$
\Delta_{2}^{*}=\delta_{3} \quad \Delta_{1}^{*}=\frac{\left(1+h_{1}^{2}\right) \delta_{3}+\left(1+h_{1}^{2}+h_{2}^{2}\right)}{\left(g_{1}^{2}+g_{2}^{2}\right) \delta_{3}-\left(1+h_{2}^{2}\right)}
$$

Proof: See Appendix B 


\section{B. Solution for the Symmetric N-Relay Network}

We consider the symmetric case where $h_{i}=h$ and $g_{i}=g$ for all $i=1, \ldots, N$. By symmetry, we have the optimal distortion level $\Delta_{i}=\Delta$ for all $i=1, \ldots, N$, and the optimization problem becomes

$$
R_{\mathrm{QMF}, \mathrm{G}}=\max _{\Delta \geq 0} \min _{k \in[0: N]} R_{k}(\Delta),
$$

where $R_{k}(\Delta):=\log \left(1+(N-k) \frac{h^{2}}{1+\Delta}\right)+\log \left(1+k g^{2}\right)-$ $k \log \left(\frac{1+\Delta}{\Delta}\right)$. By plotting the $R_{k}(\Delta)$ 's for $k \in[0: N]$ as a function of $\Delta$ for various $N, h$ and $g$ combinations, one can observe that the max min optimum appears to occur at the value of $\Delta$ where the curves $R_{0}(\Delta)$ and $R_{N}(\Delta)$ intersect. A demonstrative plot is shown for a 5-relay network in Fig. 2. It turns out that this observation can indeed be proved, for which the following lemmas are necessary:

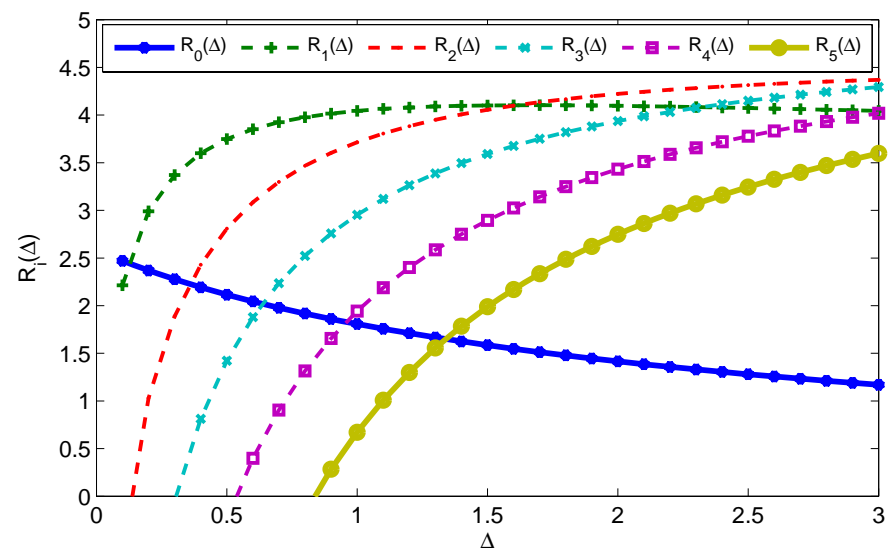

Fig. 2. $R_{i}(\Delta)$ 's as a function of $\Delta$ for a 5-Relay symmetric network with $|h|=1$ and $|g|=3.16$.

Lemma 2: There exists exactly one positive $\Delta=\Delta_{i j}^{*}$ satisfying $R_{i}(\Delta)=R_{j}(\Delta) \quad \forall i, j \in[0: N]$ and $i \neq j$.

Proof: See Appendix $C$

Lemma 3: $\lim _{\Delta \rightarrow 0}\left\{R_{i}(\Delta)-R_{i+1}(\Delta)\right\} \geq 0 \quad \forall i \in[0:$ $N-1]$.

Proof: See Appendix D

Lemma 4: Let $\Delta_{(i)(i+1)}^{*}$ be the unique positive solution of $R_{i}(\Delta)=R_{i+1}(\Delta) \quad \forall i \in[0: N-1]$. Then, $\Delta_{(i)(i+1)}^{*}$ is non-decreasing in $i$, i.e., $\Delta_{01}^{*} \leq \Delta_{12}^{*} \leq \ldots \leq \Delta_{(N-1)(N)}^{*}$.

Proof: See Appendix E

These lemmas ensure that each $R_{k}(\Delta), k \in[1: N]$ intersects $R_{0}(\Delta)$ before (in the order of increasing $\Delta$ ) any other curve. Since $R_{0}(\Delta)$ is a decreasing function of $\Delta$, the initial observation is validated and can be crystalized as follows:

Theorem 2: $\left(R_{0}\left(\Delta_{0 N}^{*}\right), \Delta_{0 N}^{*}\right)$ is the optimum ratedistortion pair, where $\Delta_{0 N}^{*}$ is the root of $R_{0}(\Delta)=R_{N}(\Delta)$.

\section{NUMERICAL EVALUATIONS}

\section{A. Achievable Rates for Static Channels}

The performance of noise-level QMF, universal QMF (derived in Sec. II-C following the spirit of [3]), and distortionoptimized QMF for canonical channel configurations of the 2-relay network is depicted in Fig. 3. In Fig. 3(a), where the multiple-access cut is the mincut, i.e., $\Omega_{\min }=\{1,2\}$, we observe that the noise-level scheme is $3 \mathrm{bits} / \mathrm{sec} / \mathrm{Hz}$ away from the cutset upper bound and the distortion optimization provides a significant improvement of $2 \mathrm{bits} / \mathrm{sec} / \mathrm{Hz}$ over the noise-level scheme. This can be attributed to the fact that in the corresponding QMF rate expression for such settings, the quantizer loss from each of the relays add up to hit the performance negatively, and this degradation is the most sensitive to quantizer distortions. Besides, since the universal QMF is derived for optimizing the worst-case gap, which occurs when the multiple-access cut is the mincut, it provides a gain over noise-level QMF. In the setting where the broadcast cut is the mincut, i.e., $\Omega_{\min }=\emptyset$ (Fig. 3(b)), the noiselevel scheme does not suffer as much (within $1 \mathrm{bit} / \mathrm{sec} / \mathrm{Hz}$ of the cutset bound) since the achievable rate is the least sensitive to quantizer distortions. Even in this case however, the optimization provides close to a $1 \mathrm{bit} / \mathrm{sec} / \mathrm{Hz}$ gain, and closely follows the cutset bound. The universal QMF performs worse since the worst-case gap does not occur when the broadcast cut is the mincut. For the asymmetric setting, where one of the cross cuts is the mincut, i.e., $\Omega_{\min }=\{1\}$, the results in Fig. 3(c) show that the noise-level scheme is approximately $1.5 \mathrm{bits} / \mathrm{sec} / \mathrm{Hz}$ away from the cutset bound, and the gap is almost completely bridged by optimizing the distortions.

Fig. 4 shows the performance of the QMF schemes over 6 -relay diamond networks. For symmetric settings, in Fig. 4(a) and 4(b) we depicted the achievable rates of noise-level QMF and the distortion-optimized QMF. We observe that in the former case where the multiple-access cut is the mincut, i.e., $\Omega_{\min }=\{1,2, \ldots, N\}$, the noise-level QMF utilizing all 6 relays achieve a rate that is more than $8 \mathrm{bits} / \mathrm{sec} / \mathrm{Hz}$ away from the cutset, while the gap from the cutset bound for the distortion-optimized QMF achievable rate is roughly 3 bits/sec/Hz. To compensate the inferior performance of noiselevel QMF, however, one can turn off a certain number of relays and get better performance. The reason is that, with fewer relays, the aggregate quantizer loss (the main source of the gap from cutset bound) is reduced. With just two noise-level QMF relays, the achievable rate is more than $2 \mathrm{bits} / \mathrm{sec} / \mathrm{Hz}$ better than using 6 noise-level QMF relays. However, reduction in the number of relays does not help if we find the optimal distortion. In the latter case where the broadcast cut is the mincut, i.e., $\Omega_{\min }=\emptyset$, we plot both the distortion-optimized QMF rates and noise-level QMF rates with relay selection. We observe that using just 4 relays turns out to be the best option for noise-level QMF, whereby it achieves within $1 \mathrm{bit} / \mathrm{sec} / \mathrm{Hz}$ of the distortion-optimized QMF rate and 2 bits $/ \mathrm{sec} / \mathrm{Hz}$ of the cutset bound. This approach follows the work in [7], where it was observed that the gap to the min-cut upper bound can be optimized by appropriately selecting the number of active relays.

We also plot the probability densities of gaps from the cutset bound for the QMF schemes over the 2-relay network (Fig. 5), where each channel coefficient $h_{1}, h_{2}, g_{1}, g_{2}$ is picked independently and uniformly at random from the linear range 


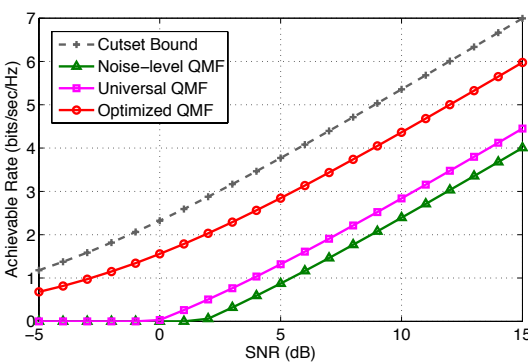

(a) $\left|h_{1}\right|,\left|h_{2}\right|=\sqrt{100 \mathrm{SNR}} ;\left|g_{1}\right|,\left|g_{2}\right|=\sqrt{\mathrm{SNR}}$

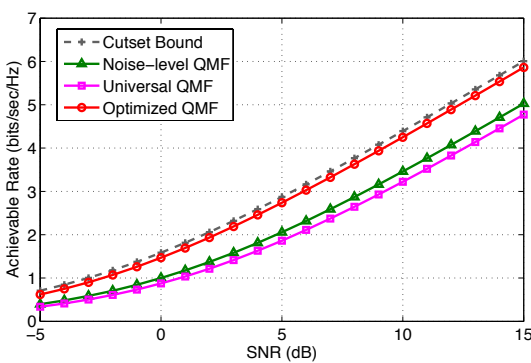

(b) $\left|h_{1}\right|,\left|h_{2}\right|=\sqrt{\mathrm{SNR}} ;\left|g_{1}\right|,\left|g_{2}\right|=\sqrt{100 \mathrm{SNR}}$

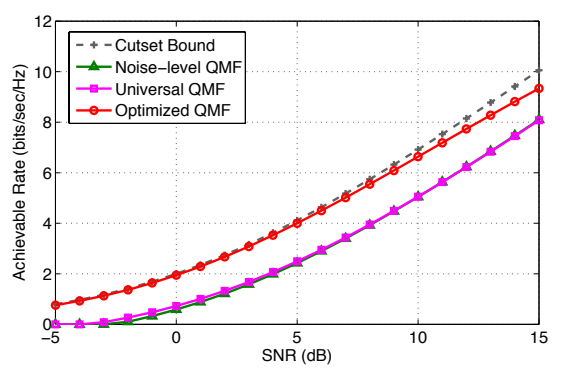

(c) $\left|h_{1}\right|,\left|g_{2}\right|=\sqrt{\mathrm{SNR}} ;\left|h_{2}\right|,\left|g_{1}\right|=\sqrt{100 \mathrm{SNR}}$

Fig. 3. QMF achievable rates for certain canonical configurations of the 2-Relay network

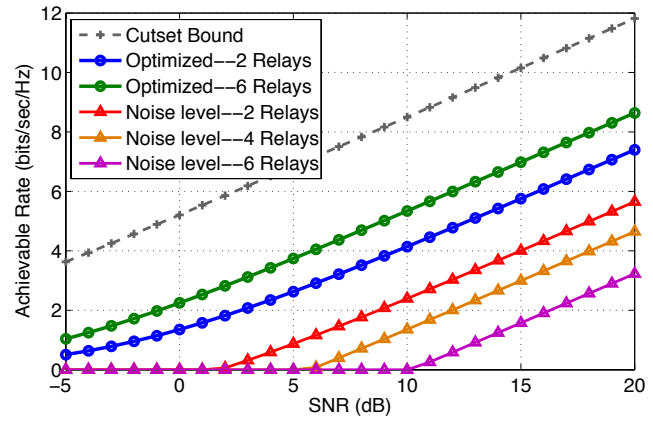

(a) $|h|=\sqrt{10 \mathrm{SNR}} ;|g|=\sqrt{\mathrm{SNR}}$

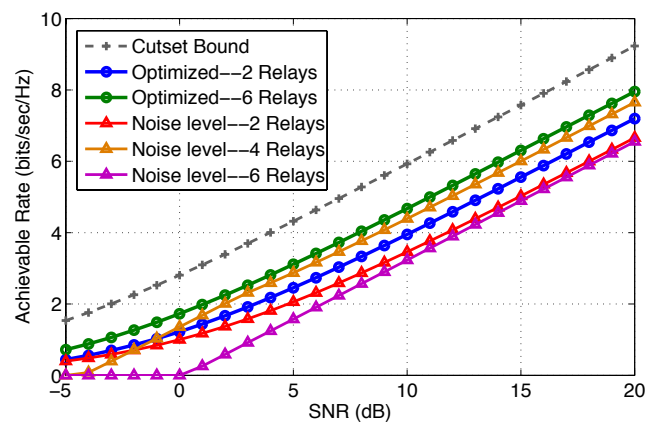

(b) $|h|=\sqrt{\mathrm{SNR}} ;|g|=\sqrt{10 \mathrm{SNR}}$

Fig. 4. QMF achievable rates for 6-Relay symmetric networks. The configuration in (b) illustrates the fact that with noise-level quantization, the achievable rate is not a monotonic function of the number of relays selected.

$[0.32,10]$. Such a range corresponds to an operating SNR in $[-10 \mathrm{~dB}, 20 \mathrm{~dB}]$. We separate the results mincut-wise for a clearer picture. The spiky nature of the distributions for the noise-level scheme reflect the fact that the quantizer loss does not adapt to the channel coefficients. Fig. 5 also demonstrates that the worst-case gap of the optimized scheme is less than 2 bits/s/Hz-an improvement of over $1 \mathrm{bit} / \mathrm{s} / \mathrm{Hz}$ over the worstcase noise-level QMF performance.

\section{B. Achievable Rates for Fading Channels}

Due to the universality of QMF operation, i.e., the encoding and decoding schemes are independent of the topology as well as the network state, QMF is an attractive cooperative communication protocol in wireless channels, where robustness to channel fading is key. In our evaluations, we consider blockfading channels where the source $S$ does not have access to

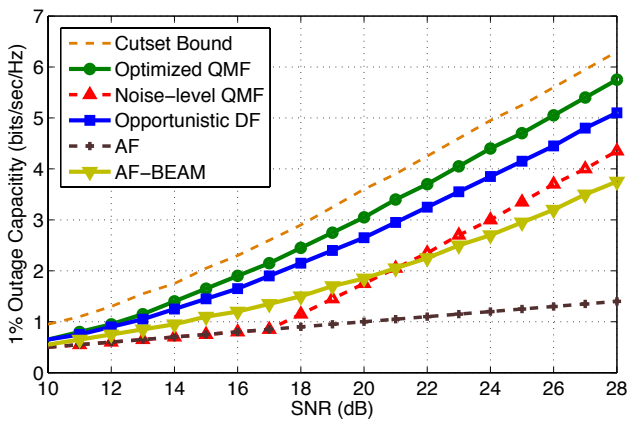

(a) $h_{1}, h_{2}, g_{1}, g_{2} \sim$ i.i.d $\mathcal{C N}(0, \mathrm{SNR})$

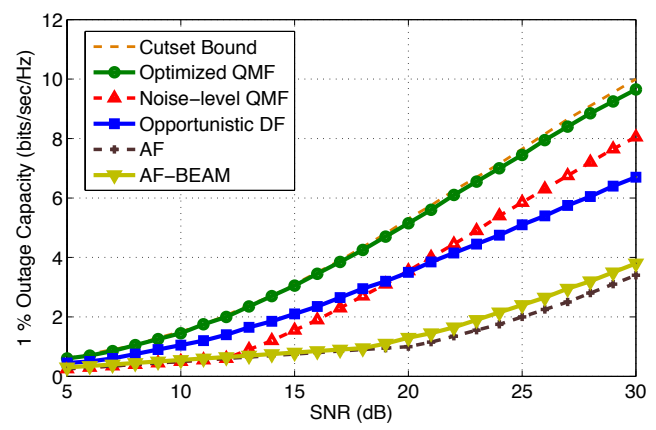

(b) $h_{1}, g_{2} \sim$ i.i.d $\mathcal{C N}(0, \mathrm{SNR}) ; h_{2}, g_{1} \sim$ i.i.d $\mathcal{C N}(0,100$ SNR $)$

Fig. 6. $1 \%$ Outage Capacity of the 2-Relay network under block-fading

the network-state information. In such settings, the appropriate metric to consider is the outage probability, defined as the probability that the channel realizations of the network will not support a given transmission rate chosen by the source.

In the absence of transmit CSI at the relays, noise-level quantization gives QMF a consistent edge over AmplifyForward (AF). In fact, in certain settings, as shown in Fig. 6(a) and 6(b), even noise-level QMF beats Amplify-Forward with beamforming (AF-BEAM) and Opportunistic DecodeForward [5]-schemes that require transmit CSI for coherent phase-combining and best-path selection respectively. In the presence of global CSI, quantizer-optimized QMF significantly outperforms the other cooperative diversity schemes, namely AF-BEAM and Opportunistic DF, and is within $0.5 \mathrm{bits} / \mathrm{sec} / \mathrm{Hz}$ of the cutset bound. In this sense, it is seen to utilize CSI in a more efficient manner vis-à-vis other schemes. 


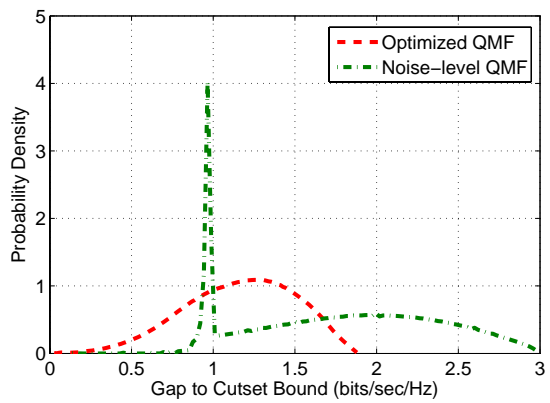

(a) $\Omega_{\min }=\emptyset$

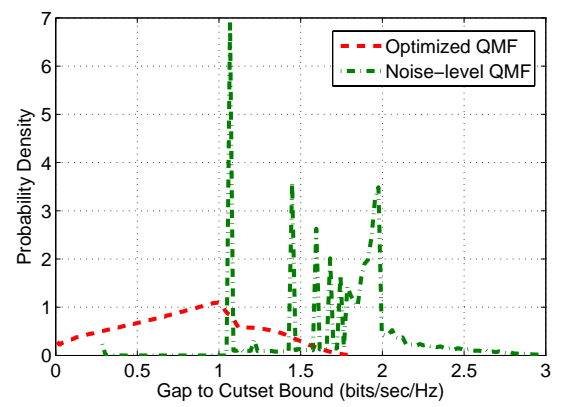

(b) $\Omega_{\min }=\{1\}$

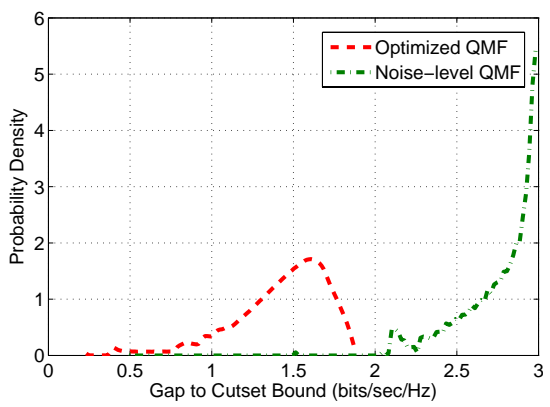

(c) $\Omega_{\min }=\{1,2\}$

Fig. 5. Distributions of gaps to cutset bound for the 2-Relay Network

\section{REFERENCES}

[1] A. S. Avestimehr, S. N. Diggavi and D. Tse, "Wireless network information flow: a deterministic approach", IEEE Trans. on Info. Theory, vol. 57, no. 4, pp. 1872-1905, Apr. 2011.

[2] A. Ozgur and S N. Diggavi, "Approximately achieving Gaussian relay network capacity with lattice codes", Proc. of IEEE ISIT 2010, Austin, Texas, pp 669-673, June 2010.

[3] S. H. Lim, Y.-H. Kim, A. El Gamal, S.-Y. Chung, "Noisy network coding", IEEE Trans. on Infor. Theory, vol. 57, no. 5, pp. 3132-3152, May 2011.

[4] U. Niesen, S. N. Diggavi, "The approximate capacity of the Gaussian $N$-relay diamond network", Arxiv E-Print, August 2010, http://arxiv.org/ abs/1008.3813

[5] A. Bletsas, A. Khisti, D. P. Reed, A. Lippman, "A simple cooperative diversity method based on network path selection", IEEE Journal on Selected Areas in Communication, vol. 24, no. 3, pp. 659-672, Mar. 2006.

[6] A. Sengupta, S. Brahma, A. Ozgur, C. Fragouli, S. Diggavi, "Graphbased codes for quantize-map-and-forward relaying", Proc. of IEEE Info. Theory Workshop (ITW), 2011

[7] C. Nazaroglu, A. Ozgur, and C. Fragouli, "Wireless network simplification: the Gaussian N-relay diamond network", in Proc. of the IEEE Int. Symposium on Inform. Theory, St Petersburg, 2011.

\section{APPENDiX A}

\section{PROOF OF LEMMA 1}

Throughout this proof we work under a fixed $\Delta_{2}$. Hence for notational convenience, let us drop the $\Delta_{2}$ argument in $R\left(\Omega ; \Delta_{1}, \Delta_{2}\right)$.

First observe that

$$
\begin{aligned}
& R\left(\emptyset ; \Delta_{1}\right):=\log \left(1+\frac{h_{1}^{2}}{1+\Delta_{1}}+\frac{h_{2}^{2}}{1+\Delta_{2}}\right) \\
& R\left(2 ; \Delta_{1}\right):=\left[\begin{array}{l}
\log \left(1+g_{2}^{2}\right)+\log \left(1+\frac{h_{1}^{2}}{1+\Delta_{1}}\right) \\
-\log \left(\frac{1+\Delta_{2}}{\Delta_{2}}\right)
\end{array}\right]^{+}
\end{aligned}
$$

are decreasing functions of $\Delta_{1}$, while

$$
\begin{aligned}
R\left(1 ; \Delta_{1}\right) & :=\left[\begin{array}{l}
\log \left(1+g_{1}^{2}\right)+\log \left(1+\frac{h_{2}^{2}}{1+\Delta_{2}}\right) \\
-\log \left(\frac{1+\Delta_{1}}{\Delta_{1}}\right)
\end{array}\right]^{+} \\
R\left(1,2 ; \Delta_{1}\right) & :=\left[\begin{array}{l}
\log \left(1+g_{1}^{2}+g_{2}^{2}\right)-\log \left(\frac{1+\Delta_{1}}{\Delta_{1}}\right) \\
-\log \left(\frac{1+\Delta_{2}}{\Delta_{2}}\right)
\end{array}\right]^{+}
\end{aligned}
$$

are increasing functions of $\Delta_{1}$. Besides, within the two curves $R\left(1 ; \Delta_{1}\right)$ and $R\left(1,2 ; \Delta_{1}\right)$, one will be no less than the other throughout all positive $\Delta_{1}$. Therefore to simplify the problem, let us first find the condition for one of them to be the dominant one, and for the remaining one can focus on the relations of the other two decreasing functions with this dominant increasing curve.

$$
\begin{aligned}
& R\left(1 ; \Delta_{1}\right) \leq R\left(1,2 ; \Delta_{1}\right) \\
\Longleftrightarrow & \log \left(1+g_{1}^{2}\right)+\log \left(1+\frac{h_{2}^{2}}{1+\Delta_{2}}\right)-\log \left(\frac{1+\Delta_{1}}{\Delta_{1}}\right) \\
& \leq \log \left(1+g_{1}^{2}+g_{2}^{2}\right)-\log \left(\frac{1+\Delta_{1}}{\Delta_{1}}\right)-\log \left(\frac{1+\Delta_{2}}{\Delta_{2}}\right) \\
\Longleftrightarrow & \left(1+g_{1}^{2}\right)\left(1+h_{2}^{2}+\Delta_{2}\right) \leq\left(1+g_{1}^{2}+g_{2}^{2}\right) \Delta_{2} \\
\Longleftrightarrow & \Delta_{2} \geq \frac{\left(1+g_{1}^{2}\right)\left(1+h_{2}^{2}\right)}{g_{2}^{2}}=\delta_{2}
\end{aligned}
$$

Below we discuss in two difference cases based on the above condition.

\section{A. $\Delta_{2} \geq \delta_{2}$}

In this case we have

$$
R_{\mathrm{QMF}, \mathrm{G}}^{*}\left(\Delta_{2}\right)=\max _{\Delta_{1}>0} \min \left\{R\left(1 ; \Delta_{1}\right), R\left(\emptyset ; \Delta_{1}\right), R\left(2 ; \Delta_{1}\right)\right\}
$$

Note that at the two extreme values of $\Delta_{1}$,

$$
\begin{aligned}
& R\left(1 ; \Delta_{1}=0\right)=0 \\
& R\left(1 ; \Delta_{1}=\infty\right)=\log \left(1+g_{1}^{2}\right)+\log \left(1+\frac{h_{2}^{2}}{1+\Delta_{2}}\right) \\
& R\left(\emptyset ; \Delta_{1}=0\right)=\log \left(1+h_{1}^{2}+\frac{h_{2}^{2}}{1+\Delta_{2}}\right) \\
& R\left(\emptyset ; \Delta_{1}=\infty\right)=\log \left(1+\frac{h_{2}^{2}}{1+\Delta_{2}}\right) \\
& R\left(2 ; \Delta_{1}=0\right)=\left[\begin{array}{l}
\log \left(1+g_{2}^{2}\right)+\log \left(1+h_{1}^{2}\right) \\
-\log \left(\frac{1+\Delta_{2}}{\Delta_{2}}\right)
\end{array}\right]^{+} \\
& R\left(2 ; \Delta_{1}=\infty\right)=\left[\log \left(1+g_{2}^{2}\right)-\log \left(\frac{1+\Delta_{2}}{\Delta_{2}}\right)\right]^{+}
\end{aligned}
$$

We conclude that $R\left(1 ; \Delta_{1}\right)$ and $R\left(\emptyset ; \Delta_{1}\right)$ will always intersect at some positive $\Delta_{1}$, and using the monotonicity of these curves, the intersection occurs at only one point. On the 
other hand $R\left(1 ; \Delta_{1}\right)$ and $R\left(2 ; \Delta_{1}\right)$ may not. Moreover, using the monotonicity of these curves, we have

$$
\begin{gathered}
\forall \Delta_{1}>0, R\left(1 ; \Delta_{1}\right) \leq R\left(2 ; \Delta_{1}\right) \\
\Longleftrightarrow R\left(1 ; \Delta_{1}=\infty\right) \leq R\left(2 ; \Delta_{1}=\infty\right) \\
\Longleftrightarrow \log \left(1+g_{1}^{2}\right)+\log \left(1+\frac{h_{2}^{2}}{1+\Delta_{2}}\right) \\
\leq \log \left(1+g_{2}^{2}\right)-\log \left(\frac{1+\Delta_{2}}{\Delta_{2}}\right) \\
\Longleftrightarrow \Delta_{2} \geq \frac{\left(1+g_{1}^{2}\right)\left(1+h_{2}^{2}\right)}{g_{2}^{2}-g_{1}^{2}} \text { and } g_{2}^{2}>g_{1}^{2}
\end{gathered}
$$

Hence, if $g_{2}^{2}>g_{1}^{2}$ and $\Delta_{2} \geq \frac{\left(1+g_{1}^{2}\right)\left(1+h_{2}^{2}\right)}{g_{2}^{2}-g_{1}^{2}}$, then $\Delta_{1}^{*}=$ $\Delta_{1}^{*}(1 ; \emptyset):=$ the intersection of $R\left(1 ; \Delta_{1}\right)$ and $R\left(\emptyset ; \Delta_{1}\right)$ :

$$
\Delta_{1}^{*}(1 ; \emptyset):=\frac{\left(1+h_{1}^{2}\right) \Delta_{2}+\left(1+h_{1}^{2}+h_{2}^{2}\right)}{g_{1}^{2}\left(\Delta_{2}+\left(1+h_{2}^{2}\right)\right)}
$$

Otherwise, we use the monotonicity of these curves to arrive at $\Delta_{1}^{*}=\min \left\{\Delta_{1}^{*}(1 ; \emptyset), \Delta_{1}^{*}(1 ; 2)\right\}$, where $\Delta_{1}^{*}(1 ; 2):=$ the intersection of $R\left(1 ; \Delta_{1}\right)$ and $R\left(2 ; \Delta_{1}\right)$ :

$$
\Delta_{1}^{*}(1 ; 2):=\frac{\left(1+g_{1}^{2}\right)\left(1+h_{1}^{2}\right) \Delta_{2}}{\left(g_{1}^{2}-g_{2}^{2}\right) \Delta_{2}+\left(1+g_{1}^{2}\right)\left(1+h_{2}^{2}\right)}
$$

Next we introduce the following claim, the proof of which is at the end of this section.

Claim 1: Within the range $\Delta_{2} \geq \delta_{2}$, we always have $\Delta_{1}^{*}(1 ; 2)>\Delta_{1}^{*}(1 ; \emptyset)$.

Therefore, $\Delta_{1}^{*}=\Delta_{1}^{*}(1 ; \emptyset)$ is the optimal solution for $\Delta_{2} \geq$ $\delta_{2}$, and $\Omega^{*}=\{1\}$ or $\Omega^{*}=\emptyset$.

\section{B. $\Delta_{2}<\delta_{2}$}

In this case we have

$R_{\mathrm{QMF}, \mathrm{G}}^{*}\left(\Delta_{2}\right)=\max _{\Delta_{1}>0} \min \left\{R\left(1,2 ; \Delta_{1}\right), R\left(\emptyset ; \Delta_{1}\right), R\left(2 ; \Delta_{1}\right)\right\}$

Again, note that at the two extreme values of $\Delta_{1}$,

$$
\begin{aligned}
& R\left(1,2 ; \Delta_{1}=0\right)=0 \\
& R\left(1,2 ; \Delta_{1}=\infty\right)=\left[\log \left(1+g_{1}^{2}+g_{2}^{2}\right)-\log \left(\frac{1+\Delta_{2}}{\Delta_{2}}\right)\right]^{+} \\
& R\left(\emptyset ; \Delta_{1}=0\right)=\log \left(1+h_{1}^{2}+\frac{h_{2}^{2}}{1+\Delta_{2}}\right) \\
& R\left(\emptyset ; \Delta_{1}=\infty\right)=\log \left(1+\frac{h_{2}^{2}}{1+\Delta_{2}}\right) \\
& R\left(2 ; \Delta_{1}=0\right)=\left[\log \left(1+g_{2}^{2}\right)+\log \left(1+h_{1}^{2}\right)\right]^{+} \\
& R\left(2 ; \Delta_{1}=\infty\right)=\left[\log \left(\frac{1+\Delta_{2}}{\Delta_{2}}\right)\right. \\
& \text { log } \left.\left(1+g_{2}^{2}\right)-\log \left(\frac{1+\Delta_{2}}{\Delta_{2}}\right)\right]^{+}
\end{aligned}
$$

We conclude that $R\left(1,2 ; \Delta_{1}\right)$ and $R\left(2 ; \Delta_{1}\right)$ will always intersect at some positive $\Delta_{1}$, and using the monotonicity of these curves, the intersection occurs at only one point. On the other hand $R\left(1,2 ; \Delta_{1}\right)$ and $R\left(\emptyset ; \Delta_{1}\right)$ may not. Moreover, using the monotonicity of these curves, we have

$$
\forall \Delta_{1}>0, R\left(1,2 ; \Delta_{1}\right) \leq R\left(\emptyset ; \Delta_{1}\right)
$$

$$
\begin{gathered}
\Longleftrightarrow R\left(1,2 ; \Delta_{1}=\infty\right) \leq R\left(\emptyset ; \Delta_{1}=\infty\right) \\
\Longleftrightarrow \log \left(1+g_{1}^{2}+g_{2}^{2}\right)-\log \left(\frac{1+\Delta_{2}}{\Delta_{2}}\right) \\
\quad \leq \log \left(1+\frac{h_{2}^{2}}{1+\Delta_{2}}\right) \\
\Longleftrightarrow \Delta_{2} \leq \frac{1+h_{2}^{2}}{g_{1}^{2}+g_{2}^{2}}
\end{gathered}
$$

Hence, if $\Delta_{2} \leq \frac{1+h_{2}^{2}}{g_{1}^{2}+g_{2}^{2}}$, then $\Delta_{1}^{*}=\Delta_{1}^{*}(1,2 ; 2):=$ the intersection of $R\left(1,2 ; \Delta_{1}\right)$ and $R\left(2 ; \Delta_{1}\right)$ :

$$
\Delta_{1}^{*}(1,2 ; 2):=\frac{\left(1+g_{2}^{2}\right)\left(1+h_{1}^{2}\right)}{g_{1}^{2}}
$$

Otherwise, we use the monotonicity of these curves to arrive at $\Delta_{1}^{*}=\min \left\{\Delta_{1}^{*}(1,2 ; \emptyset), \Delta_{1}^{*}(1,2 ; 2)\right\}$, where $\Delta_{1}^{*}(1,2 ; \emptyset):=$ the intersection of $R\left(1,2 ; \Delta_{1}\right)$ and $R\left(\emptyset ; \Delta_{1}\right)$ :

$$
\Delta_{1}^{*}(1,2 ; \emptyset):=\frac{\left(1+h_{1}^{2}\right) \Delta_{2}+\left(1+h_{1}^{2}+h_{2}^{2}\right)}{\left(g_{1}^{2}+g_{2}^{2}\right) \Delta_{2}-\left(1+h_{2}^{2}\right)}
$$

Below we derive the necessary and sufficient condition for $\Delta_{1}^{*}(1,2 ; \emptyset) \geq \Delta_{1}^{*}(1,2 ; 2)$ :

$$
\begin{aligned}
& \Delta_{1}^{*}(1,2 ; \emptyset) \geq \Delta_{1}^{*}(1,2 ; 2) \\
\Longleftrightarrow & g_{1}^{2}\left(\left(1+h_{1}^{2}\right) \Delta_{2}+\left(1+h_{1}^{2}+h_{2}^{2}\right)\right) \\
& \geq\left(1+g_{2}^{2}\right)\left(1+h_{1}^{2}\right)\left(g_{1}^{2}+g_{2}^{2}\right)\left(\Delta_{2}-\left(1+h_{2}^{2}\right)\right) \\
\Longleftrightarrow & \Delta_{2} \leq \frac{\left(1+g_{1}^{2}+g_{2}^{2}\right)\left(1+h_{1}^{2}+h_{2}^{2}\right)+\left(1+g_{2}^{2}\right) h_{1}^{2} h_{2}^{2}}{g_{2}^{2}\left(1+g_{1}^{2}+g_{2}^{2}\right)} \\
= & \delta_{1}
\end{aligned}
$$

The following claim concludes the discussion of this case. Claim 2: For any nonzero $\left\{h_{1}, h_{2}, g_{1}, g_{2}\right\}$,

$$
\frac{1+h_{2}^{2}}{g_{1}^{2}+g_{2}^{2}}<\delta_{1}<\delta_{2}
$$

Therefore, for $0<\Delta_{2} \geq \delta_{1}, \Delta_{1}^{*}=\Delta_{1}^{*}(1,2 ; 2)$ is the optimal solution, and $\Omega^{*}=\{1\}$ or $\Omega^{*}=\{2\}$. For $\delta_{1} \leq \Delta_{2}<\delta_{2}, \Delta_{1}^{*}=\Delta_{1}^{*}(1,2 ; \emptyset)$ is the optimal solution, and $\Omega^{*}=\{1,2\}$ or $\Omega^{*}=\emptyset$.

Combining the above two cases for $\Delta_{2} \geq \delta_{2}$ and $\Delta_{2}<\delta_{2}$, we have the complete characterization of $R_{\mathrm{QMF}, \mathrm{G}}^{*}\left(\Delta_{2}\right)$.

\section{Proof of Claim 1}

Assume the contrary, that $\Delta_{1}^{*}(1 ; 2) \leq \Delta_{1}^{*}(1 ; \emptyset)$. After some manipulations, we have

$$
\begin{aligned}
& \Delta_{1}^{*}(1 ; 2) \leq \Delta_{1}^{*}(1 ; \emptyset) \Longleftrightarrow \\
& \left\{\begin{array}{c}
\left(1+h_{1}^{2}\right) g_{2}^{2}\left(1+g_{1}^{2}\right) \Delta_{2}^{2} \\
+\left[\begin{array}{c}
\left(1+h_{1}^{2}+h_{2}^{2}\right)\left(g_{2}^{2}-g_{1}^{2}\right) \\
+\left(g_{1}^{2} g_{2}^{2}-1\right)\left(1+h_{1}^{2}\right)\left(1+h_{2}^{2}\right)
\end{array}\right] \Delta_{2} \\
-\left(1+g_{1}^{2}\right)\left(1+h_{2}^{2}\right)\left(1+h_{1}^{2}+h_{2}^{2}\right)
\end{array}\right\} \leq 0
\end{aligned}
$$

Denote this quadratic function of $\Delta_{2}$ by $f\left(\Delta_{2}\right)$. Now lets plug in $g_{2}^{2} \Delta_{2} \geq\left(1+g_{1}^{2}\right)\left(1+h_{2}^{2}\right)$ to give a lower bound on $f\left(\Delta_{2}\right)$ :

$f\left(\Delta_{2}\right)$ 
$\geq g_{1}^{2}\left[\left(1+h_{1}^{2}\right)\left(1+h_{2}^{2}\right)\left(2+g_{1}^{2}+g_{2}^{2}\right)-\left(1+h_{1}^{2}+h_{2}^{2}\right)\right] \Delta_{2}$

$>0$

which leads to contradiction.

\section{Proof of Claim 2}

First of all,

$$
\begin{aligned}
& \delta_{1}<\delta_{2} \\
\Longleftrightarrow & \frac{\left(1+g_{1}^{2}+g_{2}^{2}\right)\left(1+h_{1}^{2}+h_{2}^{2}\right)+\left(1+g_{2}^{2}\right) h_{1}^{2} h_{2}^{2}}{g_{2}^{2}\left(1+g_{1}^{2}+g_{2}^{2}\right)\left(1+h_{1}^{2}\right)} \\
& <\frac{\left(1+g_{1}^{2}\right)\left(1+h_{2}^{2}\right)}{g_{2}^{2}} \\
\Longleftrightarrow & \left(1+g_{1}^{2}+g_{2}^{2}\right)\left(1+h_{1}^{2}+h_{2}^{2}\right)+\left(1+g_{2}^{2}\right) h_{1}^{2} h_{2}^{2} \\
& <\left(1+g_{1}^{2}+g_{2}^{2}\right)\left(1+h_{1}^{2}\right)\left(1+g_{1}^{2}\right)\left(1+h_{2}^{2}\right) \\
\Longleftrightarrow & \left(1+g_{2}^{2}\right) h_{1}^{2} h_{2}^{2}<\left(1+g_{1}^{2}+g_{2}^{2}\right)\left(1+g_{1}^{2}\right) h_{1} h_{2}^{2} \\
\quad & \quad+g_{1}^{2}\left(1+g_{1}^{2}+g_{2}^{2}\right)\left(1+h_{1}^{2}+h_{2}^{2}\right)
\end{aligned}
$$

which is obviously true since $\left(1+g_{2}^{2}\right)<\left(1+g_{1}^{2}+g_{2}^{2}\right)<$ $\left(1+g_{1}^{2}+g_{2}^{2}\right)\left(1+g_{1}^{2}\right)$.

Second,

$$
\begin{aligned}
& \frac{1+h_{2}^{2}}{g_{1}^{2}+g_{2}^{2}}<\delta_{1} \\
& \Longleftrightarrow g_{2}^{2}\left(1+g_{1}^{2}+g_{2}^{2}\right)\left(1+h_{1}^{2}\right)\left(1+h_{2}^{2}\right) \\
&<\left(g_{1}^{2}+g_{2}^{2}\right)\left(1+g_{1}^{2}+g_{2}^{2}\right)\left(1+h_{1}^{2}+h_{2}^{2}\right) \\
& \quad+\left(g_{1}^{2}+g_{2}^{2}\right)\left(1+g_{2}^{2}\right) h_{1}^{2} h_{2}^{2} \\
& \Longleftrightarrow g_{2}^{2}\left(1+g_{1}^{2}+g_{2}^{2}\right) h_{1}^{2} h_{2}^{2} \\
&< g_{1}^{2}\left(1+g_{1}^{2}+g_{2}^{2}\right)\left(1+h_{1}^{2}+h_{2}^{2}\right) \\
& \quad+\left(g_{1}^{2}+g_{2}^{2}\right)\left(1+g_{2}^{2}\right) h_{1}^{2} h_{2}^{2},
\end{aligned}
$$

which is obviously true since $g_{2}^{2}\left(1+g_{1}^{2}+g_{2}^{2}\right)<\left(g_{1}^{2}+g_{2}^{2}\right)(1+$ $\left.g_{2}^{2}\right)$.

\section{APPENDIX B \\ PROOF OF THEOREM 1}

By the characterization in Lemma 1, (5) can be solved by finding the optimal solution in each of the above three ranges $\mathcal{I}_{1}, \mathcal{I}_{2}, \mathcal{I}_{3}$ of $\Delta_{2}$ analytically, and then find the maximum of these three.

For $\Delta_{2} \in \mathcal{I}_{3}$, note that $\Delta_{1}^{*}$ is an increasing function of $\Delta_{2}$ and that $R\left(\emptyset ; \Delta_{1}, \Delta_{2}\right)$ decreases when both $\Delta_{1}$ and $\Delta_{2}$ increase. Hence we conclude that $R_{\mathrm{QMF}, \mathrm{G}}^{*}\left(\Delta_{2}\right)$ is a decreasing function in this range. Hence

$$
\Delta_{2}^{*}=\delta_{2} \quad \Delta_{1}^{*}=\frac{\left(1+h_{1}^{2}\right) \delta_{2}+\left(1+h_{1}^{2}+h_{2}^{2}\right)}{g_{1}^{2}\left(\delta_{2}+\left(1+h_{2}^{2}\right)\right)}
$$

in this range.

For $\Delta_{2} \in \mathcal{I}_{1}$, note that $\Delta_{1}^{*}$ does not depend on $\Delta_{2}$ and that $R\left(1,2 ; \Delta_{1}, \Delta_{2}\right)$ increases when $\Delta_{2}$ increases. Hence we conclude that $R_{\mathrm{QMF}, \mathrm{G}}^{*}\left(\Delta_{2}\right)$ is an increasing function in this range. Hence

$$
\Delta_{2}^{*}=\delta_{1} \quad \Delta_{1}^{*}=\frac{\left(1+g_{2}^{2}\right)\left(1+h_{1}^{2}\right)}{g_{1}^{2}}
$$

in this range.

For $\Delta_{2} \in \mathcal{I}_{2}$, unlike the previous two cases, $R_{\mathrm{QMF}, \mathrm{G}}^{*}\left(\Delta_{2}\right)$ may not be monotone in this case. Here

$$
\begin{aligned}
& R_{\mathrm{QMF}, \mathrm{G}}^{*}\left(\Delta_{2}\right)=\log \left(1+\frac{h_{1}^{2}}{1+\Delta_{1}^{*}}+\frac{h_{2}^{2}}{1+\Delta_{2}}\right) \\
& =\log \left(1+g_{1}^{2}+g_{2}^{2}\right)-\log \left(\frac{1+\Delta_{1}^{*}}{\Delta_{1}^{*}}\right)-\log \left(\frac{1+\Delta_{2}}{\Delta_{2}}\right)
\end{aligned}
$$

The derivative of the above function with respect to $\Delta_{2}$ has the same sign as the quadratic function $q\left(\Delta_{2}\right):=A \Delta_{2}^{2}+B \Delta_{2}+C$, where

$$
\begin{aligned}
& A=h_{1}^{2}\left(1+h_{1}^{2}\right)-h_{2}^{2}\left(1+h_{1}^{2}+g_{1}^{2}+g_{2}^{2}\right) \\
& B=2 h_{1}^{2}\left(1+h_{1}^{2}\right) \quad C:=h_{1}^{2}\left(1+h_{1}^{2}+h_{2}^{2}\right)
\end{aligned}
$$

If $A \geq 0$, then the above quadratic is always positive, implying that $R_{\mathrm{QMF}, \mathrm{G}}^{*}\left(\Delta_{2}\right)$ is an increasing function. Therefore

$$
\begin{aligned}
\Delta_{2}^{*} & =\delta_{2} \\
\Delta_{1}^{*} & =\frac{\left(1+h_{1}^{2}\right) \delta_{2}+\left(1+h_{1}^{2}+h_{2}^{2}\right)}{\left(g_{1}^{2}+g_{2}^{2}\right) \delta_{2}-\left(1+h_{2}^{2}\right)} \\
& =\frac{\left(1+h_{1}^{2}\right) \delta_{2}+\left(1+h_{1}^{2}+h_{2}^{2}\right)}{g_{1}^{2}\left(\delta_{2}+\left(1+h_{2}^{2}\right)\right)}
\end{aligned}
$$

is the optimal solution.

If $A<0$, since $q(0)=C>0$ and $q(\infty)<0$, it has a only one positive root

$$
\delta_{3}:=\frac{-B-\sqrt{B^{2}-4 A C}}{2 A}
$$

If $\delta_{3} \in \mathcal{I}_{3}$ then

$$
\Delta_{2}^{*}=\delta_{3} \quad \Delta_{1}^{*}=\frac{\left(1+h_{1}^{2}\right) \delta_{3}+\left(1+h_{1}^{2}+h_{2}^{2}\right)}{\left(g_{1}^{2}+g_{2}^{2}\right) \delta_{3}-\left(1+h_{2}^{2}\right)}
$$

is the optimal solution.

If $\delta_{3}<\delta_{1}$, that is, $\delta_{3} \in \mathcal{I}_{1}$, then $q\left(\Delta_{2}\right)<0$ for $\Delta_{2} \in \mathcal{I}_{3}$ and hence $R_{\mathrm{QMF}, \mathrm{G}}^{*}\left(\Delta_{2}\right)$ is a decreasing function of $\Delta_{2}$ in this range. Therefore,

$$
\begin{aligned}
\Delta_{2}^{*} & =\delta_{1} \\
\Delta_{1}^{*} & =\frac{\left(1+h_{1}^{2}\right) \delta_{1}+\left(1+h_{1}^{2}+h_{2}^{2}\right)}{\left(g_{1}^{2}+g_{2}^{2}\right) \delta_{1}-\left(1+h_{2}^{2}\right)} \\
& =\frac{\left(1+g_{2}^{2}\right)\left(1+h_{1}^{2}\right)}{g_{1}^{2}}
\end{aligned}
$$

is the optimal solution.

If $\delta_{3} \geq \delta_{2}$, that is, $\delta_{3} \in \mathcal{I}_{3}$, then $q\left(\Delta_{2}\right)>0$ for $\Delta_{2} \in \mathcal{I}_{3}$ and hence $R_{\mathrm{QMF}, \mathrm{G}}^{*}\left(\Delta_{2}\right)$ is an increasing function of $\Delta_{2}$ in this range. Therefore,

$$
\begin{aligned}
\Delta_{2}^{*} & =\delta_{2} \\
\Delta_{1}^{*} & =\frac{\left(1+h_{1}^{2}\right) \delta_{2}+\left(1+h_{1}^{2}+h_{2}^{2}\right)}{\left(g_{1}^{2}+g_{2}^{2}\right) \delta_{2}-\left(1+h_{2}^{2}\right)} \\
& =\frac{\left(1+h_{1}^{2}\right) \delta_{2}+\left(1+h_{1}^{2}+h_{2}^{2}\right)}{g_{1}^{2}\left(\delta_{2}+\left(1+h_{2}^{2}\right)\right)}
\end{aligned}
$$

is the optimal solution. 


\section{APPENDIX C}

PROOF OF LEMMA 2

Without loss of generality, let $N \geq i>j \geq 0$. Now, the solutions to $R_{i}(\Delta)=R_{j}(\Delta)$ will satisfy

$$
\begin{aligned}
& \log _{2}\left\{\left(1+\frac{(N-i) h^{2}}{1+\Delta}\right)\left(1+i g^{2}\right)\left(\frac{\Delta}{1+\Delta}\right)^{i}\right\} \\
& =\log _{2}\left\{\left(1+\frac{(N-j) h^{2}}{1+\Delta}\right)\left(1+j g^{2}\right)\left(\frac{\Delta}{1+\Delta}\right)^{j}\right\}
\end{aligned}
$$

Raising both sides of the above equation to the power of 2 , we see that the solutions to $R_{i}(\Delta)=R_{j}(\Delta)$ are the roots of a polynomial $f(\Delta)$ which, on simplification, is as follows:

$$
\begin{aligned}
& f(\Delta)= \\
& \left(1-\frac{1+j g^{2}}{1+i g^{2}}\right) \Delta^{i-j+1}+ \\
& \left(1+(N-i) h^{2}-\left(\frac{1+j g^{2}}{1+i g^{2}}\right)\left(1+(N-j) h^{2}+i-j\right)\right) \Delta^{i-j} \\
& \left.-\left(\frac{1+j g^{2}}{1+i g^{2}}\right) \sum_{p=1}^{i-j-1}\left\{\begin{array}{c}
i-j \\
p
\end{array}\right)\left(1+(N-j) h^{2}\right)+\left(\begin{array}{c}
i-j \\
p-1
\end{array}\right)\right\} \Delta^{p} \\
& -\left(\frac{1+j g^{2}}{1+i g^{2}}\right)\left(1+(N-j) h^{2}\right)
\end{aligned}
$$

We note that in $f(\Delta)$, the coefficient of $\Delta^{i-j+1}$ is positive and that the coefficients of $\Delta_{\{p \in[0: i-j-1]\}}^{p}$ are negative. The coefficient of $\Delta^{i-j}$ may be positive or negative, depending on the channel configurations. Either way, irrespective of whether the coefficient of $\Delta^{i-j}$ is positive or negative, the number of sign changes of the coefficients of $f(\Delta)$ when written in descending order of powers is exactly 1 . By the Descartes' sign scheme, the number of positive roots of such a polynomial equation is given by $\alpha-2 m$, where $\alpha$ is the number of sign changes and $m$ is a positive integer. Since $f(\Delta)$ has exactly 1 sign change, $f(\Delta)$ has exactly 1 positive root, which proves the lemma.

\section{APPENDIX D}

\section{PROOF OF LEMMA 3}

For $i \in[0: N-1]$,

$$
\begin{aligned}
& R_{i}(\Delta)-R_{i+1}(\Delta)= \\
& \log _{2}\left\{\left(1+\frac{(N-i) h^{2}}{1+\Delta}\right)\left(1+i g^{2}\right)\left(\frac{\Delta}{1+\Delta}\right)^{i}\right\}- \\
& \log _{2}\left\{\left(1+\frac{(N-i-1) h^{2}}{1+\Delta}\right)\left(1+(i+1) g^{2}\right)\left(\frac{\Delta}{1+\Delta}\right)^{i+1}\right\}
\end{aligned}
$$

The above can be simplified as,

$$
\begin{aligned}
& R_{i}(\Delta)-R_{i+1}(\Delta)= \\
& \log _{2}\left(\frac{1+\Delta}{\Delta}\right)+\log _{2}\left(\frac{1+\Delta+(N-i) h^{2}}{1+\Delta+(N-i-1) h^{2}}\right)+\log _{2}\left(\frac{1+i g^{2}}{1+(i+1) g^{2}}\right)
\end{aligned}
$$

From the above, we note that for finite $h$ and $g$, $\lim _{\Delta \rightarrow 0}\left\{R_{i}(\Delta)-R_{i+1}(\Delta)\right\}=+\infty$ due to the presence of the term $\log _{2}\left(\frac{1+\Delta}{\Delta}\right)$ in the expression for $R_{i}(\Delta)-R_{i+1}(\Delta)$, which proves the lemma.

\section{APPENDIX E \\ PROOF OF LEMMA 4}

We define the sequence of polynomials $f_{m}(\Delta)=$ $\frac{(1+\Delta)^{m+2}}{\Delta^{m}}\left(2^{R_{m+1}(\Delta)}-2^{R_{m}(\Delta)}\right)$. Further, let $\Delta_{m}^{*}$ denote the unique positive root of $f_{m}(\Delta)$ (which is also the unique positive solution of $R_{m}(\Delta)=R_{m+1}(\Delta)$ ). Then, $\Delta_{m}^{*}$ satisfies

$$
\begin{aligned}
& g^{2} \Delta_{m}^{* 2} \\
& +\left(g^{2}\left(1+(N-m) h^{2}\right)-1-h^{2}-g^{2} h^{2}+2 m g^{2}\right) \Delta_{m}^{*} \\
& -\left(1+m g^{2}\right)\left(1+(N-m) h^{2}\right)=0
\end{aligned}
$$

For the required condition to hold true, i.e, $\Delta_{m}^{*} \leq \Delta_{m+1}^{*}$, we must have $f_{m+1}\left(\Delta_{m}^{*}\right)<0$, since $R_{m+1}(\Delta)>R_{m+2}(\Delta)$ for $\Delta \in\left(0, \Delta_{m+1}^{*}\right)$ by the preceding lemma. For $f_{m+1}\left(\Delta_{m}^{*}\right)<0$, we obtain

$\Delta_{m}^{*} \geq \frac{h^{2}\left(1+(m+1) g^{2}\right)-g^{2}\left(1+(N-m) h^{2}\right)}{g^{2}\left(2+h^{2}\right)}=\Delta_{t}$ say.

What now remains to be shown in order for the lemma to hold is that $f_{m}\left(\Delta_{t}\right)<0$ for all values of $h, g, N$ and all $m \in[0: N-1]$. Substituting the value of $\Delta_{t}$, we obtain,

$$
\begin{aligned}
& f_{m}\left(\Delta_{t}\right)=-\frac{h^{6}}{\left(2+h^{2}\right)^{2}}[\underbrace{\left.\begin{array}{c}
m^{2} g^{2}+m\left(2+(3-2 N) g^{2}\right)+ \\
g^{2}(N-1)^{2}-(N-2)+\frac{1}{g^{2}}
\end{array}\right]-}_{\Gamma} \\
& \frac{h^{4}}{\left(2+h^{2}\right)^{2}}\left[N+2(m+1)+g^{2}\left(N^{2}+2 m-1\right)+\frac{2}{g^{2}}\right]- \\
& \frac{h^{2}}{\left(2+h^{2}\right)^{2}}\left[2 N+3+4 m+g^{2}\left(4 m^{2}+4 m+(2 N-1)\right)+\frac{2}{g^{2}}\right] \\
& -\frac{1}{\left(2+h^{2}\right)^{2}}\left[2+g^{2}\right]
\end{aligned}
$$

From the above expression for $f_{m}\left(\Delta_{t}\right)$, it is clear that $\Gamma>0$ is a sufficient condition for $f_{m}\left(\Delta_{t}\right)<0$. $\Gamma$ can be viewed as a quadratic in $m$ with a discriminant $D_{\Gamma}=(5-4 N) g^{4}+4(1-$ $N) g^{2}$. Since the coefficient of $m^{2}$ is positive, the condition for $\Gamma>0 \quad \forall \quad m, g, h, N$ is $D_{\Gamma}<0$, which is satisfied for $N \geq 2$. This concludes the proof of the lemma. 